\title{
ARTIGO
}

do https://doi.org/10.22481/praxisedu.v16i39.5325

\section{LA MEDIACIÓN DE LOS PROCESOS LÓGICO-MATEMÁTICOS EN NIÑOS EN EDAD PREESCOLAR: UN ESTUDIO DE CASO}

\author{
THE MEDIATION OF THE LOGICAL-MATHEMATICAL PROCESSES IN CHILDREN \\ IN PRE-SCHOOL AGE: A CASE STUDY
}

\section{A MEDIAÇÃO DOS PROCESSOS LÓGICO-MATEMÁTICOS EM CRIANÇAS DE IDADE PRÉ-ESCOLAR: UM ESTUDO DE CASO}

\author{
María Margarita Villegas \\ Universidad Federal Rural do Semi Árido - Brasil \\ Fredy Enrique González \\ Universidade Federal do Rio Grande do Norte - Brasil
}

\begin{abstract}
Abstract: This paper studies the characterization of the mediation for the processes of the development of mathematical thinking in children of a Venezuelan public preschool. The research methodology was field-descriptive, in which participant observation and dialogue recorded by video was used. - recordings and teaching journals. The data collected belongs to 5 children of 5 and 6 years of age with their 2 teachers, which was analyzed with reference to Piaget, Vygotsky, Freire, Habermas and professionals of mathematics education for children. It is revealed that the mediation allowed to appreciate the mathematical thought subscribed by the children associated to the classification and seriation stages; It is necessary to carry out longer investigations in order to favor the knowledge of children's thinking.
\end{abstract}

Keywords: Logic-mathematical processes; Classification; Seriation; Mediation; Preschool education.

Resumen: En este trabajo se pretende caracterizar la mediación para los procesos del desarrollo del pensamiento matemático de niños de un preescolar público venezolano. La metodología de investigación fue de campo-descriptiva, en la cual se utilizó la observación participante y el diálogo registrado mediante video-grabaciones y diarios docentes. La data recolectada pertenece a 5 niños de 5 y 6 años de edad con sus 2 docentes, la cual fue analizada con referencia a postulados de Jean Piaget, Lev Vigotsky, Paulo Freire, Jürgen Habermas y profesionales de la educación matemática infantil. Se revela que la mediación permitió apreciar el pensamiento matemático suscrito por los niños asociados a estadios de clasificación y seriación; observándose necesario realizar investigaciones de mayor duración a fin de favorecer el conocimiento del pensamiento infantil.

Palabras clave: Procesos lógico-matemáticos; Clasificación; Seriación; Mediación; Educación preescolar. 
Resumo: Este artigo pretende caracterizar a mediação para os processos de desenvolvimento do pensamento matemático em crianças de uma pré-escola pública venezuelana. A metodologia de pesquisa foi a descritiva de campo, utilizando-se de pesquisa participante e de diálogos gravados em vídeos e diários dos professores. Os dados coletados são de cinco crianças de 5 e 6 anos de idade com seus 2 professores, os quais foram analisadas com referência a Piaget, Vygotsky, Freire, Habermas e profissionais da Educação Matemática para crianças. É revelado que a mediação permitiu apreciar o pensamento matemático subscrito pelas crianças associado às etapas de classificação e seriação. É necessário realizar investigações mais longas para favorecer o conhecimento do pensamento das crianças.

Palavras-chave: Processos lógico-matemáticos; Classificação; Seriação; Mediação; Educação préescolar.

\section{Introducción}

Durante la Educación Inicial, y más concretamente en el Período Preescolar, es cuando se establecen las bases para el desarrollo de los procesos lógicos sobre los que se sustenta el pensamiento matemático de la persona. Por ello, es fundamental que los docentes que se desempeñan en este nivel de la educación formal posean una formación idónea tal que les permita comprender la relevancia de dichos procesos y, al mismo tiempo, les habilite para mediar su desenvolvimiento adecuado en cada uno de los niños que tienen a su cargo; sin embargo, en general, este no parece ser el caso.

En efecto, la formación de los docentes para la educación preescolar en Venezuela es escasa; ello puede ser corroborado al analizar el número de horas de clase previstas en el plan de estudios para graduarse como profesor de Educación Preescolar en la Universidad Pedagógica Experimental Libertador (1996, 2016), donde el contenido relacionado con los procesos lógico-matemáticos se examina sólo en los primeros semestres de la carrera en las asignaturas vinculadas con aspectos generales de la psicología y del desarrollo de procesos cognitivos, con un total de nueve (9) créditos de los ciento sesenta y cinco (165), apenas 5,45\% del total, contemplados a lo largo de toda la carrera. Esto ocasiona que muchos de los egresados, docentes que se desempeñan en el nivel preescolar, no poseen una formación idónea (RUIZ MORÓN, 2008) con lo cual se priva al infante de los beneficios del desarrollo integral tan necesario durante esta etapa.

Otra de las debilidades de esta formación es que es descontextualizada y algunos docentes intentan desarrollar el pensamiento lógico-matemático de los infantes tratándolos como si fueran adultos, sin prestar atención a las particularidades que se derivan de su 
subjetividad idiosincrásica (MINISTERIO DE EDUCACIÓN Y DEPORTES, 2005). Por otro lado, en las aulas de educación preescolar, tanto de escuelas públicas como privadas, es posible encontrar materiales pre-diseñados, algunos de los mismos elaborados por los propios docentes y otros fabricados por empresas comerciales, cuyo uso está enmarcado en metodologías centradas en indicar procedimientos preconcebidos sobre el tratamiento que ha de darse a ciertos conceptos matemáticos, en lugar de propiciar la implementación de actividades de indagación en torno a situaciones concretas que puedan ser abordadas creativamente por los niños pre-escolares (ROS ROMERO, 2016).

En general, en la elaboración de tales materiales no se toman en cuenta las oportunidades que el niño tiene de entrar en contacto con diversos procesos matemáticos asociados con las situaciones que se le presentan cotidianamente en su entorno próximo (ROS ROMERO, 2016), las cuales pudieran ser asumidas como instancias naturales para la construcción de las nociones lógico-matemáticas que, usadas convenientemente, podrían ayudarlos a descubrir cómo funcionan los distintos sistemas numéricos, de modo que luego sean capaces de emplearlos en el abordaje de situaciones problemáticas que pudiesen presentárseles en su vida.

Una posible explicación de la anomalía anteriormente descrita es la creencia generalizada según la cual los procesos lógico-matemáticos del infante se dan de manera espontánea; por ello, es suficiente colocar a los niños en un espacio donde cuenten con variedad de recursos materiales y estén junto a otros niños con quienes puedan relacionarse sin mediación alguna.

Si bien, estudios como los de GÁLVEZ (2000) y UNESCO (2007) confirman que los alumnos que tienen mejor desempeño en la educación primaria son quienes han cursado el nivel preescolar; en ese escenario, se revela también cuan cierto es que en la adquisición de las nociones lógico-matemáticas por los niños en edad preescolar resulta de vital importancia ampliar la formación docente, como lo indican Ros Romero (2016), para favorecer:

[...] la comprensión y la interpretación acerca de cómo los niños y las niñas descubren el sentido del conocimiento matemático y lo utilizan recurriendo a sus propias estrategias de aprendizaje, es decir, es importante aportar conocimiento relacionado con los factores cognoscitivos en este campo: cómo aprenden y piensan, así como su relación con el contexto en el que aprenden y las emociones que les suscita. (ROS ROMERO, 2016, p. 47).

Teniendo en cuenta lo antes dicho, se podría inferir que en el nivel de educación preescolar es necesario tener en cuenta que el niño no aprende por reproducción de información transmitida, sino en función de su contexto y con base en la sensibilidad que caracteriza este 
periodo de su vida predominantemente sensorio-motriz. Es decir, estando en contacto con el hecho o asunto a ser aprendido, de modo que pueda manipular, observar y expresar el sentido que le otorga a sus experiencias y vivencias. Y además, también es preciso asumir que el aprendizaje se construye a partir las acciones ejecutadas en situaciones concretas del entorno, mediadas por los intercambios comunicativos (diálogos) con otras personas (familiares, amigos, adultos cercanos, docentes) con quienes se comparten, interiorizándolas, dichas situaciones.

Tales intercambios tienen carácter lingüístico, por tanto se debe conferir la mayor importancia al lenguaje en la educación del niño. Así que el docente de educación preescolar debe utilizar con idoneidad el diálogo con los infantes, lo cual le permitirá indagar cómo van construyendo sus conocimientos y, en particular, cómo van desenvolviendo su capacidad de razonamiento lógico-matemático.

Por ende, es necesario que realice observaciones detalladas en torno al comportamiento del niño cuando está inmerso en diversas situaciones de aprendizaje, de tal modo que pueda obtener información para que el niño logre comunicar desde su subjetividad, usando sus propias expresiones, qué actividad ha realizado y cómo se ha desenvuelto en la situación de aprendizaje en la cual ha estado involucrado; así como también ayudarlo a compartir sus vivencias sentidas durante la realización de las actividades derivadas de la situación de aprendizaje en las que ha participado.

No obstante, ello no es posible en un aula tradicional donde se diseñan estrategias con contenidos descontextualizados (ROS ROMERO, 2016), y sin la posibilidad de que el niño “[...] observe, manipule, haga preguntas, explore ideas, formule hipótesis y construya argumentos que le posibiliten al infante pensar por sí y tener ideas propias [...]" (MACHADO; CARNEIRO, 2017, p. 8).

El panorama antes descrito constituyó el incentivo principal del estudio aquí reportado, mediante el cual se pretendió indagar más acerca del proceso de desenvolvimiento del pensamiento lógico-matemático del niño de preescolar relacionándolo con la mediación dialógica realizada por el docente. La pesquisa estuvo orientada por las siguientes interrogantes: (1) ¿Cómo se lleva a cabo el proceso de construcción del pensamiento lógico-matemático en los niños preescolares? (2) ¿Cómo puede el docente de preescolar convertir las experiencias de aula en oportunidades de aprendizaje a fin de facilitar el desarrollar el pensamiento lógicomatemático de los infantes? En procura de respuestas se ejecutó la pesquisa orientada al logro de los siguientes dos: 


\section{Objetivos}

1. Caracterizar los procesos lógico-matemáticos que desenvuelven los niños en su etapa de educación preescolar (5 - 6 años de edad).

2. Propiciar el desarrollo de los procesos lógico-matemáticos de niños preescolares a través de la manipulación de material didáctico concreto y la mediación dialógica del docente.

\section{Referencial Teórico - Conceptual}

Los fundamentos teóricos y conceptuales de este estudio fueron agrupados en las dos siguientes categorías: (a) Naturaleza del pensamiento matemático en la educación preescolar; (b) La mediación como proceso social de construcción de conocimientos.

\section{EI Pensamiento Matemático en Preescolar}

El pensamiento lógico matemático del niño preescolar, de acuerdo con Fernández Bravo citado por Ros Romero (2016), se alcanza cuando el infante desarrolla sus capacidades de observación, imaginación, intuición y razonamiento, siendo este último fundamental para lograr la adquisición de conceptos matemáticos. En relación con este aspecto, Alsina (2014) destaca que en sus primeros años el razonamiento del niño es informal y tiene carácter intuitivo, ya que ellos razonan a partir de lo que ellos conocen.

El asunto de los procesos lógico matemáticos del niño fue ampliamente tratado por autores como Jean Piaget (1979, 1984, 1985) y Lev Vygotsky (1977, 1979). Uno de los aportes piagetianos más significativos ha sido reconocer que es durante las etapas iníciales de desarrollo del niño cuando se sientan las bases de todo el conocimiento posterior, tanto a nivel personal y social como en relación con el lenguaje, la lectura y la escritura.

Una manifestación del desarrollo cognoscitivo del niño preescolar (2 a 7 años) es, según Piaget (1984), su ubicación en el período pre-operacional, durante el cual los niños aún no tienen la capacidad para interiorizar plenamente una operación, no hay pensamiento lógico; pero se establecen las bases para el paso hacia éste a través de la elaboración de imágenes mentales del mundo que les rodea por medio de representaciones. El carácter esencial del pensamiento lógico, afirma Piaget (1979, p. 44), es que es operatorio, es decir, centrado en la 
acción, la cual es prolongada verbalizándola, coadyuvándole para la interiorización e inserción dentro de un sistema.

Las etapas iníciales del desarrollo tanto físico como cognitivo del niño, se identifican, según Piaget e Inhelder (1976, 2002) como: (a) período sensorio-motor (0-2 años) el cual se caracteriza por la manipulación de objetos y la percepción y exploración de sus propiedades; (b) período pre-operacional (2-7 años) durante el cual se presenta un conocimiento fundamentalmente de carácter intuitivo a partir de sus percepciones y de sus experiencias. Este período está conformado por dos sub-etapas: (a-1) la pre-conceptual o simbólica (2-4 años) durante la cual el razonamiento está diferenciado por la percepción parcial del concepto así como por asociar al mismo cuestiones que pueden tener o no que ver con él; (a-2) la intuitiva (4-7 años) que se caracteriza por la influencia que en el pensamiento del niño/a tienen las percepciones inmediatas y sus propias experiencias (Ros Romero, 2016). Se considera así que el aprendizaje germina en la acción fundamentalmente simbólica, tal como ocurre con el lenguaje en el que se integran la sociedad y el sistema educativo (Ros Romero, 2016).

Por su parte, Luciano López (1999), destaca que en el desarrollo del pensamiento del niño en edad preescolar se encuentran tres tipos de conocimiento: (a) Físico, el cual se manifiesta por medio de los sentidos; (b) Social, relacionado con las normas de convivencia y de interacción; y, (c) Lógico-Matemático, que hace referencia a la actividad mental y a los cambios cualitativos que ocurren en su mente cuando procesa e interpreta la información del mundo en que vive; los conocimientos de estas tres clases le posibilitan su adaptación al medio circundante y la toma de decisiones adecuadas cuando las circunstancias así lo ameriten. En ese contexto, se reconoce que:

La comprensión del mundo físico está fuertemente influida por categorizaciones sociales que son apropiadas de un cierto contexto cultural; $y$, por tanto, las tareas de cooperación, ayuda, imitación y guía contribuyen a este proceso de apropiación. (CANDELA, 1999, p. 275).

Particularmente relevante resulta el conocimiento lógico-matemático, sobre todo por su incidencia en el proceso de construcción del concepto de número natural, el cual, de acuerdo con Dellepiane (1995) constituye "la síntesis de las operaciones de clasificación (cardinalidad) y seriación (ordinalidad), que se realizan estableciendo correspondencias entre dos o más conjuntos” (DELLEPIANE, 1995, p. 16); también Piaget e Inhelder (2002) señalan que la clasificación es una acción de agrupamiento de objetos que pone juntos aquellos que son parecidos debido a las relaciones de semejanza, diferencia, pertenencia (vínculo entre un 
elemento y la clase a la que pertenece) e inclusión (vínculo entre una subclases y la clase de la que forma parte).

Para estos autores, la clasificación pasa por tres estadios: $1^{\circ}$ ) Colecciones figurales: aquí el niño agrupa los objetos por temáticas y hace diseños reproduciendo escenas familiares; $2^{\circ}$ ) Colecciones no figurales: en este caso, agrupa los objetos por semejanzas y diferencias pero con criterios cambiantes: una vez por forma, otra por color etc.; $3^{\circ}$ ) Inclusión Jerárquica: en este caso, el niño incluirá los elementos en una clase, teniendo en cuenta la división en subclases, disposición ésta que adquiere alrededor de los siete años de edad.

La seriación por su parte, implica la posibilidad de establecer relaciones comparativas entre los elementos de un conjunto y ordenarlos según sus diferencias, en forma decreciente o creciente (PIAGET; INHELDER, 2002; CAMPECHANO COVARRUBIAS, 1992); finalmente, mediante la correspondencia término a término, el niño va adquiriendo la noción de número natural, desenvolviéndose en tres etapas: $1^{\mathrm{a}}$ ): los niños serian entre tres y cinco elementos sin línea base; $2^{\mathrm{a}}$ ) serian por ensayo y error en una sola dirección. Si se les proporciona un nuevo objeto, vuelven a empezar. No pueden seriar por los dos extremos. $3^{\mathrm{a}}$ ) serian de forma sistemática por tamaño: si les proporciona un nuevo material saben ubicarlo en el lugar adecuado. Esto lo alcanzan ya a los siete años.

Para contribuir al desarrollo de sus procesos matemáticos, tal como se ha expuesto anteriormente, el niño debe participar en experiencias concretas, constructivas y manipulativas. Este proceso, según González (1995), transcurre a lo largo de las siguientes fases: (a) Manipulativa: poner al niño en contacto con material concreto y permitirle que lo explore, lo manipule, juegue libremente con él y le de distintos usos; (b) Verbal: dar la oportunidad al niño para que exprese oralmente qué fue lo que hizo con el material y "explique", utilizando sus propias palabras y criterios, qué manipulaciones efectuó con el material que se le proporcionó; (c) Ideográfica, solicitar al niño que, mediante un gráfico o un dibujo, traduzca sus interacciones con el material concreto en ausencia de éste; y, (d) Simbólica: Aquí ya toma lugar la abstracción, y la representación mental en símbolos y signos de los contenidos matemáticos; estas cuatro fases, señala González (1995), deben cumplirse de manera sistemática y progresiva si se desea alcanzar efectividad en la adquisición de los contenidos matemáticos, y en la mayoría de los casos, se requiere una mediación idónea por parte del docente.

En el desarrollo de los infantes, es fundamental también valorar el lenguaje con el cual ellos expresan y comunican las nociones matemáticas que están elaborando durante las etapas 
iníciales de su desarrollo tanto físico como cognitivo. En ese particular, Piaget y Vigotsky coinciden en que el lenguaje se constituye en una herramienta esencial para que el infante pueda evocar inmediatamente un objeto o evento cuando escucha algún vocablo asociado con el mismo, tales como la resolución de una situación problemática, la cual según Alsina (2014) amerita pensar y también realizar acciones de manipulación, representación y verbalización; siendo ello esencial para alcanzar la solución deseada.

Por tanto, es importante que el docente de preescolar al hablar con sus alumnos les formule preguntas que les animen a construir los argumentos mediante los cuales justifican sus decisiones y conclusiones relacionadas con las actividades en las que están envueltos, por cuanto que:

El lenguaje oral y el escrito son herramientas imprescindibles, y previas al lenguaje simbólico, para desarrollar y comunicar el pensamiento matemático; cuando un niño verbaliza en voz alta sus ideas matemáticas se favorece la comprensión y la estructuración de su pensamiento, ya que para comunicarse deben organizar sus ideas. Desde la perspectiva del maestro, el hecho de permitir al niño verbalizar implica abrir una ventana que le permite "mirar" qué hay dentro de su mente. (ALSINA, 2014, p. 12).

Por ello, es trascendental diseñar actividades en las que los infantes observen diversos objetos, estimulándolos para que se hagan preguntas a fin de que se den cuenta de diferencias y semejanzas en cuanto a forma, color, material del cual están hechos; invitándolos para que verbalicen e identifiquen la cantidad de objetos que estimen están manipulando y, entre los mismos, establezcan relaciones de tamaño (más grande, más pequeño); de masa (más pesado, más liviano); y, de longitud (más largo, más corto). Adicionalmente, podría solicitárseles que los representen mediante algún tipo de ilustración elaborada por ellos; todos estos asuntos pueden ser mejor apreciados a través de los procesos de mediación planeados en el aula.

\section{La mediación docente en el desarrollo del pensamiento matemático del niño}

Vygostky (1979) es uno de los que destaca el papel mediacional del lenguaje en el desarrollo de la actividad psíquica y social de los seres humanos. De acuerdo con este autor, el lenguaje emerge a partir de la necesidad de comunicación, lo cual pone de relieve su origen social, y al interiorizarse se convierte en pensamiento. En ese particular, Ros Romero (2016), coincidiendo con Mead, resalta que "los pensamientos y las acciones son consecuencia directa del desarrollo social mediado por el lenguaje" (ROS ROMERO, 2016, p. 66),. 
Por ende, la mediación del aprendizaje se concibe como una estrategia que, de manera consciente, desarrollan adultos o pares para activar en el aprendiz procesos mentales que conlleven a la construcción de su conocimiento. De esta manera, asumiendo una perspectiva sociocultural, al aprendizaje se le concibe como un proceso socialmente mediado que propicia la construcción de conocimientos potencialmente posibles (VILLEGAS; GONZÁLEZ, 2005; RÍOS; TEJADA; SILVA, 2004).

En el caso del desarrollo del pensamiento matemático en el nivel preescolar, la mediación es fundamental; por ello, es imprescindible que el docente acompañe al niño a fin de favorecer un desarrollo adecuado de su pensamiento lógico-matemático. Esto debido a que el aprendizaje se concibe como una co-construcción de significados lingüísticamente mediados. En efecto, de acuerdo con Habermas (1981) los seres humanos disponen del lenguaje para comunicarse entre sí, lo cual hace viable el entendimiento que no es más que una socialización y negociación de significados y acuerdos compartidos. En este sentido podría decirse que el aprendizaje constituye un acto comunicativo (Habermas, 1981) en el cual se comparten interpretaciones, sentidos e intencionalidades.

"La importancia de las interacciones dialógicas basadas en la igualdad de todos los participantes en los actos comunicativos y en la valoración de los argumentos en detrimento de las posiciones de poder de las estructuras sociales" es resaltada por Ros Romero (2016, p. 66 69). En ese aspecto, Freire (1984) sostiene quela conciencia, se construye en diálogo entre lo exterior y lo interior, entre objetividad y subjetividad.

Es decir, hay que escuchar a los estudiantes para -en diálogo- construir el conocimiento, ya que el escucharlos implica dejar que ellos hablen, “...tenemos que hablarles y hablar con ellos, no hablar para ellos" (FREIRE, 1984, p. 113). Por ende, en la formación y educación en el nivel preescolar, se hace necesario desarrollar procesos de mediación dialógica que faciliten conocer cómo piensa e interpreta el niño.

En el proceso de mediación, VILLEGAS (2007) ha identificado cuatro fases: $1^{\text {a }}$ ) contacto inicial; $2^{\mathrm{a}}$ ) impacto significativo; $3^{\mathrm{a}}$ ) inmersión en la situación; y $4^{\mathrm{a}}$ ) reflexión.

Durante el contacto inicial tienen lugar aquellas interacciones preliminares, generalmente manifestadas en forma de preguntas in situ, focalizadas sobre un aspecto del objeto que se busca analizar. Esto permite que cada uno de los sujetos implicados se presten atención unos a otros, se tomen en cuenta entre sí, se escuchen y cada uno procure atender las expectativas iniciales que plantean los demás; y, con ello, favorecer ubicar el objeto de interés sobre el cual se intenta sostener la relación comunicativa 
El impacto significativo acontece cuando el sujeto ubica el objeto de interés en la situación, esta ubicación es facilitada mediante preguntas de indagación o diálogos cómplices que se orientan hacia la profundización en aquellos aspectos que han impactado sobre el actor del hecho y que tímidamente emanan de él; tiene lugar cuando se aprecia la novedad, la cual, quizás estaba cubierta por el manto del prejuicio, de la ignorancia, de la confusión. El proceso de significación del impacto comienza con la atención que prestan quienes interaccionan con el objeto, a un aspecto colateral y secundario; luego, poco a poco, van asignándole sentido hasta alcanzar un estadio en el que el objeto se hace plenamente significativo para el sujeto.

La anterior fase deriva en la inmersión en la situación. En esta fase es cuando se van develando sus aspectos característicos, típicos, originales, innovadores, que hacen posible reconocer el asunto como elemento destacado y destacable en el contexto, por su forma, color, tamaño, textura etc., y también por el papel que desempeña en la configuración de la situación; un indicio importante de su impacto es la capacidad para reconocer cómo se modificaría la situación si el objeto en consideración estuviese ausente; por ejemplo, darse cuenta de que si le faltaran dos de sus patas, una mesa de tabla rectangular no podría sostenerse.

La significación del impacto se evidencia en manifestaciones de alegría o asombro al tomar conciencia de que en el aspecto considerado reside el foco del asunto; sin embargo, esta experiencia se vive, no sólo de manera subjetiva individual, sino intersubjetivamente; en efecto, los actores [piénsese, por ejemplo, en un grupo de niños participando colectivamente después de la fase manipulativa de materiales concretos, tales como tacos, regletas, envases plásticos, bloques de lego, piezas de un juego de dominó etc.] hacen referencia verbal a su experiencia personal y vivencial con el objeto, utilizando expresiones que son mutuamente comprensibles ya que remiten a situaciones que le son comunes; esto es acompañado por la alegría de sentirse adecuadamente interpretado, es lo que pudiera denominarse como interpretación consentida.

Finalmente, el tránsito de lo concreto hacia lo abstracto que se propicia con el proceso de mediación, requiere del estímulo de la reflexión por parte del agente mediador, eventualmente el docente, quien coadyuva a la construcción de una red de relaciones entre: el objeto y su contexto; la dinámica de la situación tanto en presencia del objeto como en ausencia del mismo; las manifestaciones ostensibles y las no ostensibles del objeto, es decir, su apariencia y su esencia; de esta manera se logra una elaboración integrada que representa la totalidad de la situación, en la que cobran sentido los objetos y las operaciones realizadas entre éste y los demás elementos de la situación, haciendo viable de este modo la abstracción; es 
decir, la posibilidad de trascender lo específico y concreto y posicionarse en lo general, lo cual es crucial para la construcción de los conceptos matemáticos, en particular el de número.

\section{Método}

El estudio aquí reportado tuvo un carácter dual: documental y descriptivo. En primer lugar se realizó una revisión bibliográfica destinada a examinar los planteamientos conceptuales de algunos autores acerca del pensamiento lógico matemático del niño preescolar. Luego, se realizó un estudio de campo en el contexto natural de un aula de clase de nivel preescolar en un jardín de infancia público venezolano, donde fueron propiciadas experiencias propiciadoras del pensamiento lógico-matemático de un grupo de cinco (5) niños en edad preescolar, con edades cronológicas comprendidas entre cinco (5) años y dos (2) meses y seis (6) años y seis 6 meses. La maestra responsable del aula es graduada como docente en la especialidad de Educación Preescolar.

Para recolectar la información se ejecutaron siete sesiones de observación participante de dos horas (120 minutos) cada una, mediadas a través de diálogos intencionados entre la maestra y los niños sobre el foco de investigación: pensamiento lógico matemático; como medios de registro se utilizaron: grabadora de audio, cámara fotográfica, hojas de trabajo de los niños y diarios de anotaciones de la docente.

Durante las siete (7) sesiones de clase, se observó y acompañó a los niños cuando interaccionaban con un material didáctico concreto; en primer lugar se invitaba a los niños a que realizaran actividades sobre operaciones lógicas; luego se le formulaban preguntas para que cada niño expusiera oralmente lo que estaba haciendo y expresara los criterios o razones que usaba para asignar significado a cada situación. A medida que observaba y se dialogaba con los niños, se grababa el audio de todo lo que estaba ocurriendo y, al finalizar cada clase, se hacía un registro pormenorizado de lo que los infantes habían hecho, observado, sentido y expresado durante la clase.

La información recaudada fue transcripta y organizada en matrices elaboradas al efecto, cuyo contenido fue analizado cualitativamente teniendo en cuenta: (a) las afirmaciones relativas al nivel de desarrollo cognoscitivo de los niños en edad preescolar formuladas por Piaget y Inhelder (1976, 2002); Dellepiane (1995); Luciano López (1999); Alsina (2014) y Ros Romero (2016); (b) los planteamientos mediacionales y del lenguaje de Vygostky (1977, 1979); Habermas (1981); Freire (1984) y Villegas (2007); (c) las fases del tránsito de lo concreto a lo 
abstracto planteadas por González (1995); y, (d) las acciones de clasificación, seriación y correspondencia puestas en juego por los niños cuando realizaban las actividades constructivas y manipulativas propuestas para el trabajo con los alumnos en el aula.

En los diálogos con los niños y las niñas se usan las letras M para identificas la docente y la letra $\mathrm{N}$ para hacer referencia a los infantes con quienes se dialogan. Así mismo, como respeto a sus personas y protección de su identidad, en la imagen de cada uno de ellos se cubrió su rostro con una carita feliz y los nombre usados son seudónimos.

\section{Resultados}

En este trabajo se reportan los siguientes asuntos: (a) Fases y procesos del pensamiento matemático en que se encuentra cada uno de los niños estudiados; (b) los criterios que usaron para denominar su proceso; y, (c) los procesos de mediación docente-niño que tuvieron lugar durante el estudio realizado. En cada caso, se presenta una figura donde se muestra la representación física elaborada por la/el niña/o acompañado del diálogo sostenido por la docente/investigadora con los niños.

\section{Fases y Procesos en el desarrollo del pensamiento matemático}

A continuación, se muestran algunas evidencias de los procesos matemáticos que realizaron los niños con la mediación pertinente por parte del docente.

\section{Procesos de Clasificación}

Se les permitió a los niños explorar y manipular diversos materiales concretos; por ejemplo, se les presentó un envase contentivo de creyones y lápices de diferentes materiales y colores; luego de consentirles que los exploraran, se les pidió que los agruparan de acuerdo a como ellos creyeran que debían ir. Así mismo, se les solicitó que señalaran los criterios de acuerdo con los cuales realizaron el proceso de agrupamiento. A continuación se presentan varios de los casos con los diálogos sostenidos con los niños donde revelan que están en la fase manipulativa de las colecciones figurales hacia la clasificación operatoria (PIAGET; INHELDER, 1976). 


\section{Etapas de clasificación.}

En este caso, el infante, requiere comprender que un objeto no puede ser miembro de dos clases opuestas. Para ello, debe ser capaz de tener un criterio de clase y entender que los miembros de una clase son semejantes en algo; reconocer que una clase se puede describir enumerando todos los elementos que la componen; y, comprender el fenómeno de inclusión de clase, es decir, entender cómo se relacionan los distintos objetos a observar. Así se tienen tres etapas: 1era de colecciones figurales, de 2 a 5 años; 2 da etapa, colecciones no figurales de 5 a 7 años; y, 3era etapa, de inclusión de clase ya el niño ha logrado clasificar por más de un criterio, tales como semejanzas, diferencias, pertenencia e inclusión.

\section{Colecciones Figurales ${ }^{1}$}

Para este tipo de clasificación tenemos tres casos, expuestos a continuación

\section{$\underline{1 \text { er caso de colecciones figurales }}$}

\section{Diálogos maestra niño}

M: Por qué has colocado eso así

$\mathrm{N}$ : "Estos van juntos por que son iguales"

M: y si las organizamos así, intentando ponerlos en serie.

$\mathrm{N}$ : Noo, "Los iguales van juntos"

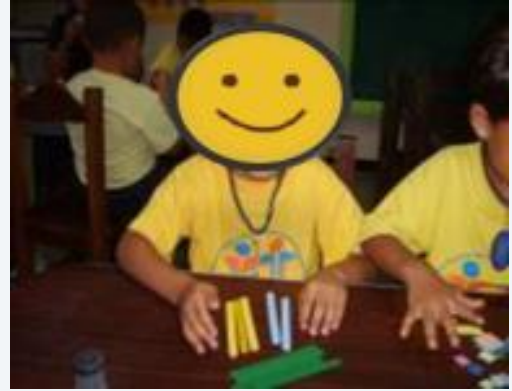

Figura 1 agrupación

\section{2do caso. Colecciones Figurales.}

\section{Diálogo maestro niña.}

M: Dayana, aquí está este envase... ¿Qué tiene adentro?

$\mathrm{N}$ : Muchos colores.

M: Bueno, pon junto lo que va juntos, por favor, colócalos en el sitio donde deben ir,

$\mathrm{N}$ : ¿Así maestra? ¿Este va con este?

M: No se mi niña, colócalos como tu creas que deben ir...

$\mathrm{N}$ : Bueno así.

M: Ok. ¿Terminaste?

$\mathrm{N}:$;Si!

M: ¿Cómo los colocaste?

$\mathrm{N}$ : Así, estos con estos.

\footnotetext{
${ }^{1}$ Los autores dejamos constancia de nuestro agradeciendo a la docente de aula, Profesora Isanic Torres MSc, quien realizó los registros de las actividades realizadas con cada infante en el aula objeto de estudio.
} 
M: ¿Y Por qué los colocaste así?

$\mathrm{N}$ : Este es del mismo color de este, el otro va con el azul porque son iguales.

Al revisar los diálogos de los dos casos anteriores, se aprecia que los criterios para colocar los objetos juntos están asociados al color: los verdes, los amarillos y azules. En consecuencia, se interpreta que estos niños se ubican en la clasificación de Colecciones Figurales, es decir, los agrupan por tanteo intuitivo, sin plan previo, por criterios de percepción más que de pertenencia a una clase y no aceptan que haya otro criterio.

\section{3er caso de Colecciones Figurales}

\section{Diálogo: maestra niña}

M: Dayana, aquí tienes este dominó, ¿Te gustaría jugar con él?

N: Si maestra yo se jugar dominó.

M: Esta bien mi reina, juega con el dominó entonces.

$\mathrm{N}$ : Mira maestra hice un edificio de uno, dos y tres pisos. (Señalando con su dedo cada uno de los pisos del "Edificio") y dice:

"Como el apartamento de mi tía".

M: Muy bien y ¿En cuál piso vive tu tía?

$\mathrm{N}$ : En este...(Señaló el 2 piso con su dedo índice)

En este 3er caso de Colecciones Figurales se les permitió manipular fichas de dominó de madera el cual contenía en cada una de sus caras, figuras de animales para relacionar; no obstante, la niña optó por agrupar los objetos en el espacio representando una figura identificada como un edificio de habitación. Este proceso se denomina colecciones figurales, los junta en una forma espacial que representa a un objeto que le es familiar, por atribuirle semejanzas con los que encuentra en su entorno inmediato o de su vida cotidiana. Se aprecia que no domina la comprensión (identificación del conjunto de características comunes de una clase) ni la extensión (saber identificar todos los objetos que pertenecen a la clase). En la mediación, a través del diálogo se perciben los criterios que usa para su la representación que elabora.

\section{Colecciones No Figurales.}

Para este tipo de clasificación se tienen tres casos 


\section{$\underline{1 e r \text { caso de colecciones No Figurales. }}$}

\section{Diálogos maestra con el mismo niño del 1er caso}

N. "Estos van juntos porque son iguales"

M y por qué no los colocas juntos a los anteriores (indicándole las paletas)

$\mathrm{N}$ : "Noo, porque las paletas no van con los triángulos"

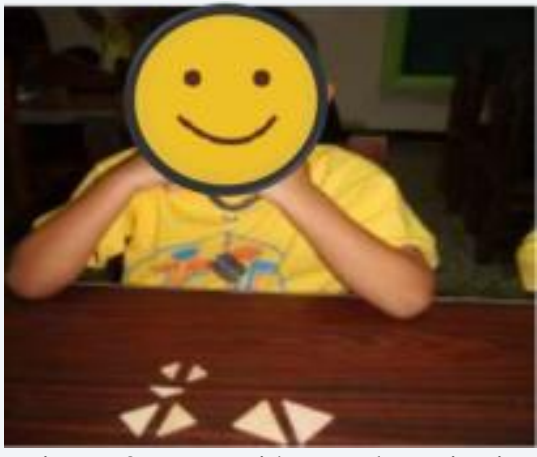

Figura 3 agrupación por dos criterios

En el anterior caso, se observa que al darle otras piezas de diferentes color y forma, los agrupa por forma y tamaño y de acuerdo a su naturaleza: figuras geométricas; es decir, los clasifica por un segundo criterio, lo cual es característico de la Colecciones No Figurales.

\section{2do caso de Colecciones No Figurales}

\section{Etapa de Clasificación: Colecciones No figurales, hacia la clasificación operatoria.}

M: Aquí tienes este envase Clara, ¿Qué N: Esta bien (comenzó a separar los cosas tiene dentro?

\section{$\mathrm{N}$ : Colores maestra y también lápices.}

M: Muy bien, ahora ¿me ayudarías a agruparlos? ¿Crees que hay manera de colocarlos? ¿Cómo deben ir?

$\mathrm{N}$ : Si mae, (Comenzó a clasificar los creyones de acuerdo con el color)

$\mathrm{N}$ : Ya terminé maestra

M: Cuéntame ¿qué hiciste?

$\mathrm{N}$ : Puse los amarillos con los amarillos, los rojos aquí con los otros rojos, los verdes juntos, los rosados y los azules.

M: ¿Ya lo terminaste? ¿Crees que podemos hacer otro grupo con el grupo que tiene más?

$\mathrm{N}$ : ¿Con este mae? (Señalando el conjunto de los creyones amarillos)

M: Si mi corazón, con ese, ¿Puedes hacer otro grupo?

N: $S i ́$

M: Hazlo entonces. creyones de cera de los de madera y de los lápices)

M: Y estos amarillos ¿por qué los separas?

$\mathrm{N}$ : Porque esos son lápices.

$\mathrm{M}:$ ¿Y estos lápices?

$\mathrm{N}$ : Ah, esos van aquí porque son grandes, no chiquitos como estos. (Los lápices estaban nuevos).

M: Muy bien princesa, te tomaré una foto y listo.

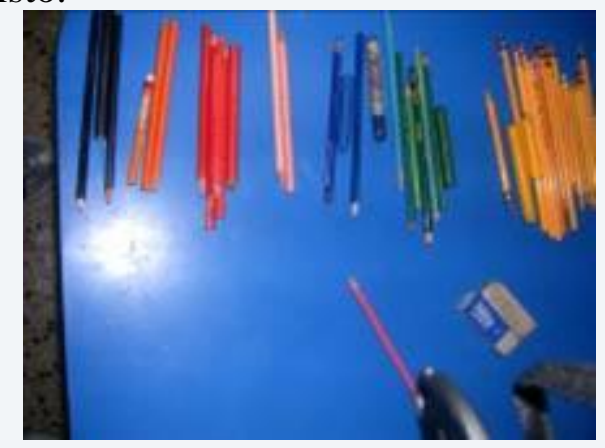

Figura 4 agrupación por dos criterios

Como se puede ver la niña Clara agrupa por un criterio de semejanza (color), y luego por la naturaleza del material (creyones de cera, de madera, etc), teniendo en cuenta la división dentro de la clase, por ende pertenece al criterio de Colecciones No Figurales ya que agrupó por dos criterios, en este caso color y material 
En todos los casos expuestos anteriormente (colecciones figurales y no figurales), se observa el fenómeno denominado comprensión (Piaget e Inhelder, 1976) por organizarlos por su cualidades comunes (relaciones de semejanza) o según la diferencias (relaciones de alteridad) ubicándoles en subclases atendiendo a su naturaleza, forma y función. Así, se observó que lo hacían sin simultaneidad, es decir sin un plan previo y con ausencia de un criterio único: los agruparon por su naturaleza: de madera, de cera; por su tamaño: grande, pequeño; por su función: lápices, creyones, entre otros.

Las experiencias de aprendizaje asociadas con materiales concretos, permitieron apreciar que con los tres niños de se dieron la fase Manipulativa y Verbal (GONZÁLEZ, 1995). En la fase manipulativa, ellos se centran en reconocer las propiedades de los objetos, lo cual les permite desarrollar su conocimiento físico, el cual al ser verbalizado, expresado a través de las palabras que nombran esos objetos, da lugar a la fase verbal, facilitándose el conocimiento social al reconocer las convenciones que se usan en el contexto cultural donde conviven (LUCIANO LÓPEZ, 1999). Esto es de suma importancia ya que las expresiones de los niños nos permiten adentrarnos en el mundo interior del infante, reconociéndose así que "el pensamiento no se expresa simplemente en palabras sino que se expresa a través de ellas" (VYGOTSKY, 1977, p. 166) y que, como lo señala Rodríguez Arocho (2006, p. 376), el funcionamiento mental es conceptualizado como un derivado del funcionamiento inter-mental que emerge por la internalización y el dominio de los procesos sociales.

\section{Proceso de Seriación}

La seriación supone que el niño comprenda que se pueden organizar los objetos en orden jerárquico, creciente o decreciente: mayor que, menor que; entendiendo la transitividad o transferencia (PIAGET; INHELDER, 2002).

\section{Estadios de seriación.}

1er caso de seriación: Etapa de Seriación:

Se les presentó un envase con paletas de maderas de diferentes formas y tamaños, se le permitió que las exploraran y las manipularan, luego les pedimos que ordenaran las paletas según el orden que debían tener. 
Diálogo maestra niña

M: Karla, toma estas paletas, míralas bien y ordénalas como deberían ir.

$\mathrm{N}$ : ¿Cómo Maestra?

M: Ponlas en el orden que tú creas.

$\mathrm{N}$ : Bueno, primero estos triángulos, los voy a poner así, listo maestra.

$\mathrm{M}$ : ¡Ah muy bien! Y ¿Cómo los ordenaste?

$\mathrm{N}$ : Este primero porque es el más

pequeñito, después este mediano y el grande.

M: ¡Muy bien! ¿Y las paletas?

$\mathrm{N}$ : También, primero la pequeña, la mediana y después la grande.

M: Está mi niña. Gracias.

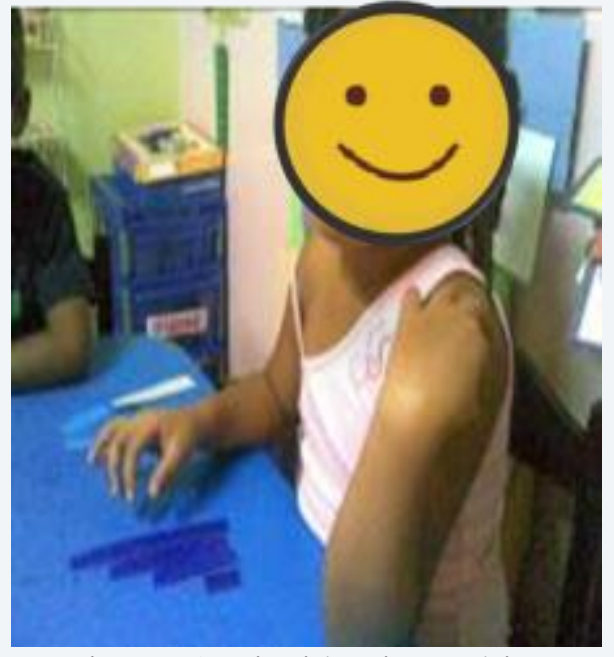

Figura 5seriación sistemática

Se observa que Karla se encuentra en la etapa de seriación del 3erestadio sistemática, pues efectúa la agrupación de forma sistemática, por tamaño, sin tanteo, comparando los dos extremos.

2do Caso de seriación: Serie de 4 o 5 Elementos con Línea Base

Se le otorgó una serie de 4 rectángulos de cartulina de color y se le pidió que los ordenara.

Diálogo: maestra niño

M: Pedro, ten estas figuras. ¿Cuál figura es esa?

N: Rectángulo.

M: Bien, y ¿esos rectángulos son todos iguales?

N: No, hay unos pequeños y unos más grandes.

M: Ah bueno, ¿Los puedes poner en orden cómo se verán mejor?

$\mathrm{N}$ : Listo maestra.

M: ¿Cómo los ordenaste? ¿Cuál colocaste primero?

$\mathrm{N}$ : Ay mae, primero va el grandote, después el otro grande, después el chiquito y el más chiquitico.

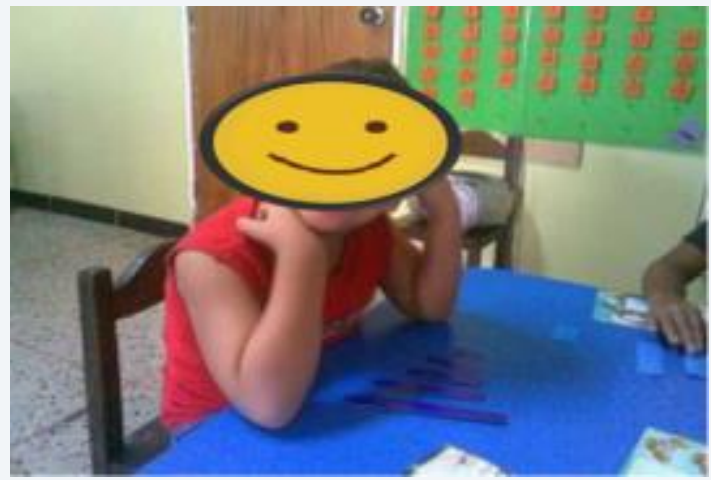

Figura 6seriación sistemática.

$\mathrm{N}$ : No mae, así no puede ir porque ese va al lado del otro pequeño.

M: Entonces la dejamos ahí.

$\mathrm{N}$ : Si así es como se ve bien.

M: ¡Que bien!. Y si agarro este chiquitico y lo coloco después del grande.

Con la actividad anterior se constata que el niño Pedro también está en el estadio 3ero de la seriación denominada sistemática, pues demuestra tener criterios de reversibilidad al no aceptar ubicar un objeto en un extremo arbitrario sino en concordancia con el tamaño que le corresponde. Es decir, compara los objetos por tamaño y teniendo en cuenta los dos extremos, pudiendo insertar un nuevo elemento sin tener que reiniciar la serie 
3er. Caso Seriación. Seria de 405 objetos con línea base superior o inferior

En este caso y también en el siguiente, se les solicitó a los niños que expresaran mediante el lenguaje oral (Fase Verbal) los procesos llevados a cabo y que describieran las representaciones realizadas. Así en una sesión de clase se les suministró una lámina con figuras de mar y con espacios en los cuales debían colocar las fichas con figuras, bien sea seriándolas o clasificándolas.

Diálogo: maestra niño

M: Daniel, ¿Qué puedes observar en estas láminas?

$\mathrm{N}$ : Animales maestra.

$\mathrm{M}$ : $\mathrm{Y}$ ¿Qué tipo de animales son?

$\mathrm{D}$ : Animales marinos.

M: Muy bien. Y ¿en estas fichas que observas?

$\mathrm{N}$ : También animales marinos.

M: ¿Se parecen entre ellos?

$\mathrm{N}$ : Si, aquí hay cangrejos que son iguales y en este dibujo también, también se parecen las estrellas y los peces.

M: Perfecto, ahora dime ¿Para qué son los espacios en blanco que tiene la lámina grande?

$\mathrm{N}$ : Bueno maestra ahí tienes que colocar las fichas más pequeñas.

M: Ah, está bien. ¿Lo harías?

$\mathrm{N}: \mathrm{Si}$.

M: Ok. Avísame en cuanto lo tengas listo.

$\mathrm{N}$ : Al término de cinco minutos, llama para comunicar lo que hizo y dice:Ya mae.

M: Ahora cuéntame ¿Cómo los organizaste?

$\mathrm{N}$ : Coleccioné los caballitos de mar. Los busqué todos y los coloque juntos aquí.

$\mathrm{M}$ : Ah muy bien. Y ¿Las demás figuras? ¿Qué puedes hacer con ellas?

$\mathrm{N}$ : Las puedes poner en otra lámina o se la das a Pedro para que coleccione otras.

$\mathrm{M}:$ ¿Te parece? Pero si yo te doy otra ficha para que la coloques en tu lámina.

$\mathrm{N}$ : No mae, no se puede porque ya no hay espacios además no se parecen a estos caballitos.

M: Ah, está bien. Entonces dejemos los caballitos en su espacio. ¿Te puedo tomar una foto?

$\mathrm{N}$ : Si mae, ¿Así, me veo bien?

$\mathrm{M}$ : Si, saliste bellísimo.

$\mathrm{N}$ : Déjame ver...

\section{Relaciones de correspondencia}

Por esta se entiende establecer una relación de vínculo que sirve de nexo. Es decir, un elemento de un conjunto se vincula con otro ya sea por un criterio establecido y observado. Esto puede ser por poseer la misma cantidad, tipo de objeto, objeto a objeto, objeto signo, objeto función, objeto parte. 
1er caso: Correspondencia articulada

Al igual que en el caso anterior, el niño debía describir oralmente las acciones realizadas (Fase Verbal). Para ello, se le suministró una lámina con figuras de mar y con espacios en los cuales debía colocar las fichas con figuras, en función de un vínculo.

Diálogo: maestra niño

N: La coloqué en donde estaba dibujado el cangrejo porque allí es donde va.

M: Ah ¿sí? Y ¿Qué pasa si yo coloco a un pez en ese sitio?

$\mathrm{N}$ : Aquí van los cangrejos, porque aquí esta dibujado el cangrejo y si lo pones en otro lado se pone bravo y te muerde.

M: ¿Me muerde? Ay que mal... ¿Y las otras figuras también tienen su lugar?

N: Si, mira, este es el lugar de la estrella (Señalando el sitio debajo del dibujo de la estrella de mar). Aquí solo debes poner estrellas. En este sitio solo puedes poner peces, aquí conchas y por aquí van los

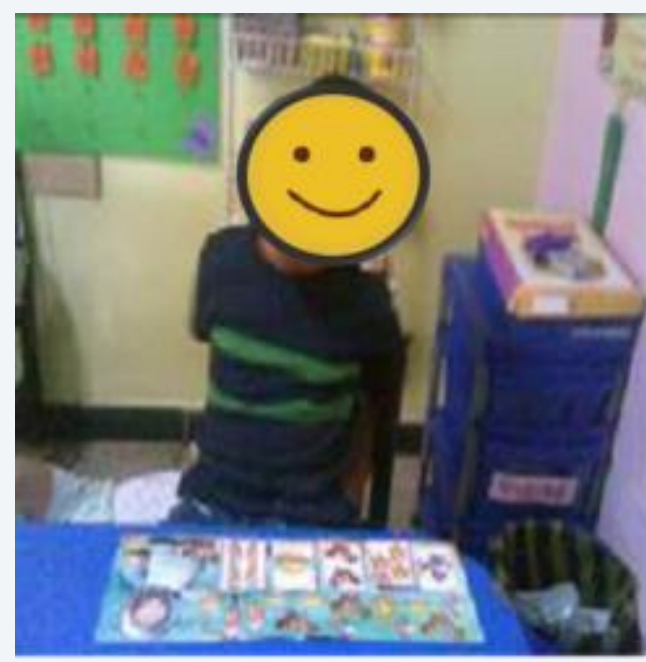

Figura 7 Correspondencia término a término caballitos de mar. (Fue señalando cada espacio debajo de la imagen correspondiente.

M: ¡Ah ya entendí! Entonces los colocaste todos en su sitio

Pedro clasificó los objetos por asociatividad y pertenencia inclusiva en una clase, lográndose la correspondencia término a término de acuerdo al tipo de objeto.

\section{2do caso de Correspondencia por función.}

Primero, se invitó a los niños a que relacionaran cada lámina con la figura correspondiente, de acuerdo con su función (ver Fig. 8).

\section{Ubica cada lámina con su figura de acuerdo con su función, según corresponda.}

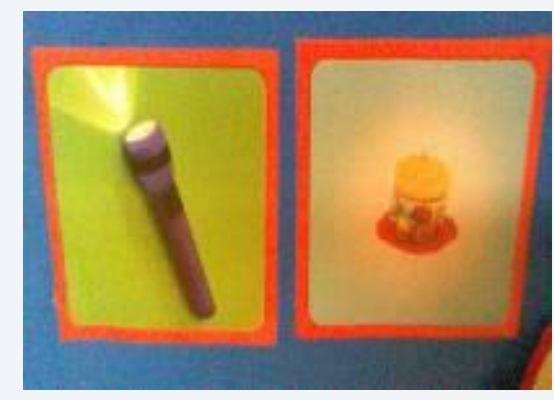

Figura 8 correspondencia por función
M:Karla ¿Qué has hecho?

$\mathrm{N}$ : "La linterna va con la vela"

M: "Y eso ¿por qué?"

$\mathrm{N}$ : "Porque las dos ayudan a ver de noche"

M:Muy bien, Clara

En este caso las maestras, como investigadoras, esperaban que la niña hiciera la correspondencia de linterna con linterna y vela con vela; pero ella actuó de manera diferente, 
lo que demuestra la importancia del diálogo, ya que éste nos permite reconocer las subjetividades del pensamiento infantil.

\section{Correspondencia de Figuras Opuestas:}

En este caso, se le pidió a Karla que representara con un dibujo (Fase Ideográfica) el proceso de correspondencia realizado.

M. Coloca las figuras con su correspondiente opuesto, luego realiza el dibujo de la actividad.

$\mathrm{N}$ : "Dibujaré la linterna prendida y la otra apagada, la vela pequeña y la grande y los tambores"

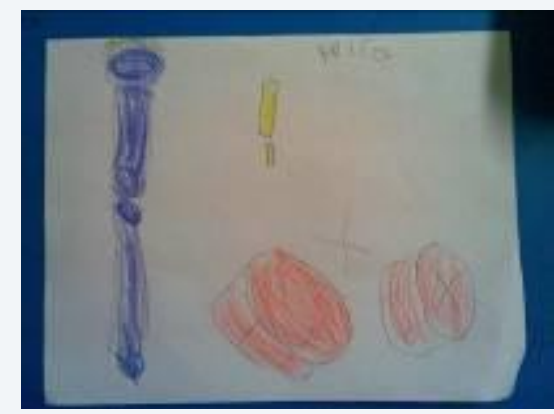

Figura 9 correspondencia por opuestos Así se ve que hace correspondencia de opuestos, según la función. Prendida y apagada.

\section{Comentarios Generales}

Como se ha podido observar en este trabajo los niños necesitan manipular el material para reconocerlo, requieren verbalizarlo para comunicar lo que saben (VYGOTSKY, 1977; ALSINA, 2014). Esto se hizo posible a través de la mediación docente, con la cual se les estimulaba para que expusieran los criterios que usaron el organizar los diversos materiales que manipulaban. Ello nos permitió propiciar el conocimiento tanto físico como social del niño. Así mismo, de manera intencional se propició que dibujaran la operación realizada a fin de conocer el pensamiento ideográfico, como indica GONZÁLEZ (1995) y de esa manera reconocer el dominio de los procesos sociales (Vygostky, 1979; VILLEGAS, 2007) de los cuales se ha hecho beneficiario

La verbalización de la experiencia permite apreciar los criterios que el niño usa para agrupar los materiales, favorecida por la mediación a través de preguntas orientadas a reconocer el proceso en el que está inmerso el niño y respetar de esta manera sus criterios. Por su parte, la comunicación ideografía, permite reconocer la competencia para evocar gráficamente las representaciones mentales que ha construido el niño y con ello promover la sistematización del pensamiento, asunto que fomentará pasar al siguiente estado de operaciones lógicas.

En todos los casos, se hace evidente la importancia de la mediación a través de las etapas que se señala VILLEGAS (2007): una, contacto inicial, cuando se les solicitaba a los niños que llevaran a cabo una determinada actividad; dos, el impacto significativo, cuando cada sujeto 
reconoce el hecho que el otro quiere exponer, comunicar; tres, la inmersión en la situación, proceso que permite la intersubjetivación, en este caso, entre las docentes investigadoras y los alumnos; y, cuatro, la reflexión, proceso que permite hacer la abstracción de las actividades realizadas, que si bien éstas se manifestaron en sólo los docentes como investigadoras, no deja de reconocerse el papel importante que tuvieron los niños estudiados y sus actividades realizadas para que ésta pudieran emerger.

\section{Conclusiones}

Los niños sujetos de estudio realizan actividades de clasificación, las cuales se pueden ubicar: una niña de 5 años, en el 1er estadio de la clasificación colecciones figurales; dos niños de 5 años con varios meses de edad se ubicaron en el 2 do estadio de colecciones no figurales; y, dos de ellos, de seis y más años, están en proceso de adquirir la clasificación operatoria y la seriación jerárquica, lo cual implica que dominan la reversibilidad y transitividad.

Se pudo constatar que la mediación del docente es importante para poder facilitarle a los niños/ñas el alcance de los procesos de conocimiento físico, social y lógico, lo cual, en el caso estudiado, brindó la oportunidad de participar en experiencias que favorecieron el aprendizaje y con ello posibilitar el tránsito a estadios posteriores. Se reconoce, así mismo, que mientras se realice mayor cantidad de actividades destinadas a desarrollar sus nociones lógicomatemáticas, mejor será la adquisición del proceso de abstracción y con ello el pensamiento lógico, por lo que se recomienda desarrollar un estudio con mayor duración (un periodo escolar completo) con el propósito de revelar con más exhaustividad las características de las etapas de construcción de las operaciones lógicas, las cuales por su nivel de complejidad demandan de condiciones de tiempo y concentración, así como competencia del docente para reconocer y mediar idóneamente los microprocesos implícitos en cada actividad tomando en cuenta la idiosincrasia de los niños estudiados.

\section{REFERENCIAS}

ALSINA, Ángel. (2014). Procesos matemáticos en Educación Infantil: 50 ideas clave. Números. Revista Didáctica de las Matemáticas, 86, pp. 5-28.

CAMPECHANO COVARRUBIA, Juan (1992). Sobre la enseñanza de las pre-operaciones lógico-matemáticas. Revista La Tarea. Revista de Educación y Cultura, n. ${ }^{\circ} 1$ Año 1992 
(Sección 47) Disponible en:

http://www.latarea.com.mx/articu/articu1/campechano1.htm.Consulta 23012000.

CANDELA, Antonia. (1999). Prácticas discursivas en el aula y calidad educativa. Revista Mexicana de Investigación Educativa, v. 4, n. 8, 273-298.

DELLEPIANE, Alicia. (1995). Matemática para la educación inicial. Buenos Aires, Argentina: Magisterio de Río de la Plata.

FREIRE, Paulo. (1984). La importancia de leer y el proceso de libración. Buenos Aires: Siglo Veintiuno editores.

GÁLVEZ, Inmaculada Egido (2000). La educación inicial en el ámbito internacional: Situación y perspectivas en Iberoamérica y en Europa. Revista Iberoamericana de Educación. Número 22. Educación inicial. Disponible: http://www.rieoei.org/rie22a06.htm

GONZÁLEZ, Fredy Enrique (1995). Algunas ideas acerca de la enseñanza de la matemática en la escuela básica. En F. E. GONZÁLEZ. La enseñanza de la matemática: proposiciones didácticas. (Capítulo 6), 37-65. Caracas: FEDUPEL.

HABERMAS, Jürgen. (1981). Teoría de la acción comunicativa I. México: Taurus.

LUCIANO LÓPEZ, Margarita. (1999). Matemática educativa para inicial y básica: aprendizaje del número natural. Santo Domingo, República Dominicana: Buho.

MACHADO, Maria Flávia Dias; CARNEIRO, Reginaldo Fernando. Percepção matemática na educação infantil a partir de brincadeiras. Trabalho de Conclusão de Curso;

(Graduação em Pedagogia) - Universidade Federal de Juiz de Fora; Orientador: Reginaldo Fernando Carneiro. Disponível em:

http://www.ufjf.br/pedagogia/files/2017/12/Percep\%C3\%A7\%C3\%A3o-matematica-naeduca \%C3\%A7\%C3\%A3o-infantil-a-partir-de-brincadeiras.pdf. Acesso: 29FEV2020A

MINISTERIO DE EDUCACIÓN Y DEPORTES (2005). Educación inicial: procesos matemáticos. República Bolivariana de Venezuela, Caracas: Editorial Noriega

PIAGET, Jean e INHELDER, Bärbel. (1976). Génesis de las estructuras lógicas elementales. Buenos Aires: Guadalupe.

PIAGET, Jean e Inhelder, Bärbel. (2002). Psicología del niño. Madrid: Morata.

PIAGET, Jean. (1979). Psicología de la inteligencia. Buenos Aires: Psique

PIAGET, Jean. (1984). La representación del mundo en el niño. Madrid: Morata

PIAGET, Jean. (1985). La construcción de lo real en el niño. Barcelona: Crítica

RÍOS, Pablo; TEJADA, Miren (de); SILVA, Antonieta (2004). Teorías vigentes sobre el desarrollo humano. Caracas: FEDEUPEL. 
RODRÍGUEZ AROCHO, Wanda. (2003). Interacción social y mediación semiótica: Herramienta para reconceptualizar la relación desarrollo y aprendizaje. Educere, (Venezuela) Año 6 (20),369-375.

ROS ROMERO, María. (2016). Pensamiento y leguaje matemático en el contexto de educación infantil: un acercamiento interpretativo. Departamento de Didáctica y Organización Escolar Universidad Complutense de Madrid. Facultad De Educación. Tesis Doctoral. Disponible en: https://eprints.ucm.es/40436/1/T38109.pdf

RUIZ MORÓN, Deyse. (2008). Las estrategias didácticas en la construcción de las nociones lógico-matemáticas en la educación inicial. Paradìgma (Venezuela), 29 (1), 91-112.

Disponível em: http://revistaparadigma.online/ojs/index.php/paradigma/article/view/401

TORRES, Isanic. (2007). Los procesos lógico matemáticos en preescolar. Trabajo de Grado de Maestría no publicado. UPEL Maracay, Venezuela

UNESCO (2007). Informe de seguimiento de la EPT en el mundo: bases sólidas. Atención y educación de la primera infancia. Paris. Francia: ONU

UNIVERSIDAD PEDAGÓGICA EXPERIMENTAL LIBERTADOR (1996, 2016). Plan de estudios de Educación Preescolar. Disponible:

http://www.ipmar.upel.edu.ve/New\%20Site/docencia/plan_est_preescolar.htm.

VIGOTSKY, Lev. (1977). Pensamiento y lenguaje: comentarios críticos de Jean Piaget. Buenos Aires: La Pléyade

VIGOTSKY, Lev. (1979). EI desarrollo de los procesos psicológicos superiores. Madrid: España: Crítica.

VILLEGAS, Ma Margarita; GONZÁLEZ, Fredy. (2005). La Construcción de Conocimiento por parte de Estudiantes de Educación Superior. Un caso de futuro docentes. Revista Perfiles (México). XXVII (109-110,) 117-139.

VILLEGAS, María Margarita. (2007). Los procesos de mediación en la enseñanza y el aprendizaje. UPEL Maracay: Material Didáctico inédito.

VILLEGAS, María Margarita; GONZÁLEZ, Fredy Enrique (2005). La Construcción de Conocimiento por parte de Estudiantes de Educación Superior. Un caso de futuro docentes. Revista Perfiles (México). XXVII (109-110,) 117-139. Disponível em: http://www.scielo.org.mx/scielo.php?script=sci_arttext\&pid=S0185-26982005000200006 


\section{SOBRE OS AUTORES:}

\section{María Margarita Villegas}

Doutora em Educação pela Universidad Pedagógica Experimental Libertador (UPEL), Venezuela. Professora Visitante da Universidade Federal Rural do Semi Árido (UFERSA) PRÓ-REITORIA DE PESQUISA E PÓS-GRADUAÇÃO - POSENSINO. E-mail: margaritavillega@hotmail.com

iD http://orcid.org/0000-0002-4965-2291

\section{Fredy Enrique González}

Doutor em Educação (com Énfase em Educação Matemática) pela Universidad de Carabobo UC (Venezuela). Professor Visitante da Universidade Federal do Rio Grande do Norte (UFRN), Centro de Educação -Departamento de Práticas Educacionais e Currículo - Programa de PósGraduação em Educação - PPGEd. E-mail: fredygonzalez@ hotmail.com

(iD) http://orcid.org/0000-0002-8079-3826 